\title{
INTRODUZIONE: OLTRE LA DICOTOMIA MANIFATTURA-SERVIZI. UN TERRITORIO ANCORA DA ESPLORARE
}

\section{di Marco Bettiol e Eleonora Di Maria}

Questo numero di Economia e società regionale nasce dall'idea di esplorare un territorio ancora poco studiato come quello della crescente convergenza tra il mondo della manifattura e quello dei servizi. Molti autori, soprattutto in passato, hanno indicato nei servizi uno spazio autonomo di crescita economica, capace di compensare la graduale perdita della manifattura a causa della crescente capacità competitività dei paesi emergenti. Questa lettura si giocava su un'ipotesi radicale: l'opposizione tra immateriale e materiale, dove il primo è di competenza dei servizi (pensiamo al software) e il secondo della manifattura. Una dicotomia che è stata particolarmente enfatizzata dalla globalizzazione che ha visto i paesi economicamente più avanzati specializzarsi in attività terziarie a maggiore valore aggiunto mentre quelli in via di sviluppo puntare sulla produzione manifatturiera a basso costo.

Questo scenario è tuttavia in profonda evoluzione. In questi ultimi anni c'è stata da più parti una progressiva crescita di attenzione verso la sfera della produzione e le attività manifatturiere e sul loro rinnovato ruolo nel processo di generazione del valore e come fonte di competitività per imprese e territori.

Da un lato, la globalizzazione spinta e l'imporsi sulla scena internazionale di paesi come la Cina - definita come la piattaforma produttiva mondiale - ha mostrato un percorso quasi irreversibile di spostamento delle attività manifatturiere dai paesi occidentali a quelli emergenti e a più basso costo del lavoro, lasciando ai primi una specializzazione sulle attività terziarie (design, marketing, R\&D). Dall'altro lato la crisi ha mostrato il ruolo che può avere la prevalenza della finanza sull'economia reale e la necessità di tornare a ridare importanza ai processi industriali rispetto a logiche finanziarie per la crescita.

Questo quadro e le sue evoluzioni future possono essere meglio comprese richiamando il concetto di terziarizzazione dell'economia (Di Bernardo, 1991). Il dibattito sulla trasformazione in chiave terziaria dell'economia è molto ampio e articolato e si fonda sull'idea di una contrapposi- 
zione tra i settori della manifattura (prodotti e processi tangibili) e del terziario (servizi intangibili) nel processo di generazione del valore e nelle dinamiche di sviluppo economico territoriale.

A una prima fase di crescita incentrata sui processi manifatturieri come elemento chiave per la competitività di imprese e territori viene contrapposta un'enfasi sulle attività terziarie come generatrici di valore, presupponendo una riconversione di professioni e imprese e nuove forme di divisione del lavoro a livello internazionale. Da un lato paesi come gli Stati Uniti specializzati in attività come la R\&D e l'innovazione sciencedriven e dall'altra paesi come la Cina o il Messico dedicati alle attività manifatturiere. Un processo che è stato ben descritto dagli studi sulle global value chains (cfr. Cattaneo et al., 2010). Adottando questa prospettiva, paesi produttori come 1'Italia - che negli anni '90 (età d'oro dei distretti industriali) occupava il $4^{\circ}$ posto a livello mondiale dopo Stati Uniti, Giappone e Germania per quota globale di valore aggiunto lordo legato al manifatturiero (McKinsey, 2012) - avrebbe dovuto riorientarsi verso i servizi, internazionalizzando le attività produttive ed accrescendo l'occupazione nel terziario. Prendendo a riferimenti i dati dello studio McKinsey sulla manifattura nel contesto globale emerge ad esempio che la Cina dal 1980 al 2010 è passata dalla $7^{a}$ alla $2^{a}$ posizione nel ranking globale sopra citato.

Allo stesso tempo, anche la sfera dei servizi ha subito una forte accelerazione, perdendo nel tempo i connotati di ambito di supporto alla produzione, a bassa produttività (morbo di Baumol) e forte connotazione locale per lasciare spazio ad un settore capace di produrre autonomamente valore. L'investimento in tecnologie dell'informazione e della comunicazione ha aumentato la produttività nei servizi. Le imprese di servizi hanno visto accrescere - in particolare in alcune specializzazioni - la propria dimensione media (si pensi alla grande distribuzione specializzata), adottando pratiche organizzative e manageriali tipiche dell'impresa manifatturiera (anche in grado di organizzare e controllare catene del valore globali). Inoltre i servizi hanno dimostrato di saper sviluppare percorsi e modelli autonomi di innovazione (Gallouj e Djellal, 2010). Hanno sviluppato percorsi di internazionalizzazione, in parte a seguito dell'internazionalizzazione manifatturiera (Miozzo e Miles, 2002). In particolare i servizi ad alto contenuto di conoscenza (knowledgeintensive business services o Kibs legati al design, alla comunicazione, alle Ict, all'engineering e $\mathrm{R} \& \mathrm{D}$, studi professionali) hanno rappresentato un ambito interessante di crescita occupazionale e di investimento delle politiche pubbliche per lo sviluppo e la competitività regionale e nazionale (Muller e Zenker, 2001). 
In realtà, a nostro avviso, lo scenario che si sta delineando è molto più ampio ed articolato di una semplice contrapposizione manifattura/servizi, con esiti non scontati anche per l'Italia. La combinazione originale tra servizi ad alto valore aggiunto e le attività manifatturiere più tradizionali sta configurando la nascita di una nuova generazione di imprese capaci di competere a livello nazionale ed internazionale. Questa trasformazione rappresenta una interessante linea di evoluzione del tradizionale modello del made in Italy e, seppure ancora minoritaria, rappresenta un fenomeno che va approfondito soprattutto nell'ottica dello sviluppo locale.

Il numero monografico che presentiamo intende mettere a fuoco tale trasformazione indagando in modo specifico il tema dei processi di innovazione e delle nuove forme di interazione tra la sfera del materiale tradizionalmente attribuita alla manifattura - e quella dell'immateriale assegnata al terziario. Ci interroghiamo sul rapporto, o meglio, sulla convergenza tra la sfera della manifattura e quella dei servizi di diversi punti di vista.

Quali differenze esistono oggi, o meglio esistono ancora differenze tra un'impresa come Decathlon - che distribuisce un ampio portafoglio di prodotti per lo sport, ma che sviluppa come gruppo Oxylane proprie linee di prodotto, ha proprie marche, brevetti e una rete di fornitori mondiale e Geox - tra i primi produttori di calzature che ha diverse decine di brevetti, un brand forte, una rete distributiva mondiale in proprietà $\mathrm{o}$ in franchising e collaborazioni di fornitura in Europa dell'Est e Far East?

Il dibattito sull'importanza della manifattura sta crescendo in questi ultimi tempi, con un focus sul ritorno alla produzione da parte di paesi come ad esempio gli Stati Uniti che da tempo avevano abbandonato la manifattura per concentrarsi sui servizi. Tuttavia non si tratta di riportare banalmente indietro attività produttive organizzate secondo logiche fordiste. Al contrario, viene molto enfatizzato un nuovo modo di organizzare la produzione in cui l'innovazione tecnologica - dalle Ict alle stampanti 3D - giocano un ruolo interessante e la sfera imprenditoriale ed artigianale (makers) hanno un peso determinante (come descritto nel contributo di Vladi Finotto in questo numero).

L'enfasi sulla terziarizzazione dell'economia ha fatto pensare allo sviluppo economico ancorato ai servizi, concentrando l'attenzione al processo di sviluppo di imprese del terziario come settore, secondo una visione settorialista dello sviluppo economico (Di Bernardo, 1991). Tuttavia, la trasformazione in chiave terziaria ha riguardato anche l'interno delle imprese manifatturiere, che in parte è stato letto in chiave di servitization (Vandermerwe e Rada, 1988). 
Proponiamo una lettura che enfatizza la complementarietà tra due attività di generazione del valore e fonte di competitività per imprese e territori, in cui non si può più generare distinzione forzata tra manifattura $\mathrm{e}$ servizi in funzione della dimensione materiale e immateriale di questi, utilizzata come uno dei primari fattori distintivi tra i due ambiti di attività economiche.

La manifattura infatti diventa immateriale (Rullani, 2004). Lo sviluppo del marketing e il valore assegnato al brand non più come elemento accessorio del prodotto, ma come asset autonomo che l'impresa può gestire (secondo Interbrand 2012, ad esempio, il valore del brand CocaCola è pari a 77.839 mila USD) mostrano uno spostamento di baricentro nel processo di generazione del valore. Tale trasformazione è stata ben descritta anche da Pine e Gilmore nel loro libro sull'economia dell'esperienza (2000). La manifattura si fa servizio. La servitization descrive infatti il processo di traslazione dal bene (tangibile) al servizio, con un orientamento verso la costruzione di relazioni di lungo termine con i propri clienti. In questa prospettiva le imprese manifatturiere adottano approcci tipici dell'impresa di servizi, promuovendo l'interazione e la costruzioni di soluzioni per il cliente.

Il contributo di Marco Paiola in questo numero è proprio incentrato sulla trasformazione dal servizio alle soluzioni, in cui Paiola evidenzia come le imprese di produzione modificano la propria offerta cercando di fornire non semplicemente beni e nemmeno solo servizi, ma soluzioni in grado di fronteggiare le richieste dei clienti e produrre valore. Vengono evidenziati in modo particolare percorsi distinti in relazione alle competenze delle imprese ed all'estensione dei servizi inclusi nell'offerta (più $o$ meno prossimi alla base manifatturiera dell'impresa).

Con un accenno diverso che lega manifattura, servizi ed internazionalizzazione, il contributo di Bortoluzzi, Chiarvesio e Tabacco si interroga sull'evoluzione delle strategie delle imprese italiane connesse al binomio manifattura - servizi nel momento in cui si confrontano con i mercati emergenti. Le nuove opportunità di mercato aprono la strada per ripensare business consolidati ancorati alla manifattura e per sviluppare un mix originale che permette di adattarsi in maniera proattiva al nuovo contesto competitivo - non basta più un buon prodotto per vendere su tali mercati.

Allo stesso tempo i servizi si industrializzano. Da un lato, come abbiamo citato prima, le imprese del terziario iniziano a sviluppare logiche gestionali ed organizzative tipiche dell'impresa manifatturiera, come l'adozione della standardizzazione in vista dei vantaggi di efficienza delle economie di scala tipiche della grande impresa manifatturiera fordista (cfr. il caso "scuola" di McDonald's nel servizio di ristorazione descritto 
da Ritzer (1997)). Il contributo di Enzo Rullani in questo numero si focalizza proprio sulla produttività nei servizi e sulle diverse forme attraverso cui le imprese di servizi possono accrescere la propria produttività, superando una contrapposizione tra visioni diverse sul tema.

Dall'altro lato le imprese di servizio iniziano ad investire sul fronte della produzione materiale, integrando al proprio interno nuove funzioni della catena del valore. Questo fenomeno è particolarmente visibile se prendiamo come riferimento le imprese che operano nel design (servizi orientati al business-to-business, Kibs). Come evidenziato nel contributo curato da noi insieme con Roberto Grandinetti, l'adozione di una classificazione settoriale per distinguere tra imprese di servizi e non tende a mostrare profondi limiti proprio in ragione dei cambiamenti che abbiamo descritto. In particolare l'osservazione sul fronte dei Kibs mostra come anche le imprese manifatturiere possano definirsi a tutti gli effetti dei Kibs nel momento in cui si concentrano su funzioni terziarie che per definizione sono tipicamente Kibs (es. R\&D, la comunicazione o il design). Allo stesso tempo, grazie alle tecnologie di rete ed alla disponibilità di competenze manifatturiere anche con livelli qualitativi adeguati nel panorama internazionale, i Kibs possono selezionare fornitori a scala globale a cui demandare la funzione di produzione dei propri prodotti.

$\mathrm{Nel}$ numero che presentiamo è anche inserito un caso aziendale - Antarei (curato da Valentina De Marchi) - che esprime bene la transizione verso un modello competitivo più complesso in cui il fare manifatturiero non è più sufficiente, occorre inserire elementi di servizio (in chiave di sostenibilità e web 2.0) per rifondare la competitività d'impresa: da fornitore di antine a "consulente" d'arredo per la cucina (con upgrading verso il prodotto finito).

Per concludere ci limitiamo a sottolineare alcune parole chiave che possono aiutare a comprendere meglio il processo di convergenza che abbiamo contribuito a delineare in questo numero.

Personalizzazione/standardizzazione: la prima non è unicamente appannaggio dei servizi e la seconda solo della manifattura. Come abbiamo mostrato la quota di personalizzazione richiesta alle imprese manifatturiere è cresciuta e queste ultime possono farvi fronte con modalità diverse - dal ricorso a soluzioni, all'investimento su prodotti "intelligenti" e versatili fino all'utilizzo di nuove tecnologie (3D) e artigianato (serie limitate, piccola scala efficiente di produzione). Allo stesso tempo anche $\mathrm{i}$ servizi standardizzano e si industrializzano orientandosi verso i vantaggi delle economie di scala.

Interazione: l'interazione con il cliente - ma anche con la rete del valore - è fondamentale nel nuovo paradigma postfordista e l'investimento 
sul fronte del Crm e delle nuove tecnologie - in un quadro di innovazione aperta e collaborativa - ha permesso anche alle imprese manifatturiere di gestire efficaci relazioni con il mercato "imparando" dal mondo dei servizi. In alcuni casi l'interazione significa anche nuova divisione del lavoro in cui è lo stesso consumatore/cliente ad essere messo "al lavoro" svolgendo attività un tempo appannaggio dell'impresa.

Internazionalizzazione: come evidenziano gli studi sui Kibs l'internazionalizzazione non riguarda unicamente attività produttive, ma anche attività di servizio siano esse direttamente connesse o meno al processo manifatturiero (es. design o R\&D). Soprattutto emerge un'internazionalizzazione non sono reattiva, ma anche proattiva delle imprese di servizi che si orientano verso la ricerca di nuovi clienti oltre confine - superando le necessità di un'interazione connessa alla prossimità - ma anche di fornitori con cui impostare relazioni produttive.

La presenza in Italia e in Veneto di imprese con connotazioni sia manifatturiere che di servizio ne fa un ambito interessante per andare a studiare nel tempo questo percorso di integrazione tra due mondi che sono stati a lungo contrapposti.

\section{Riferimenti bibliografici}

Di Bernardo B. (1991), “Dal terziario al neo-industriale”, Economia e Diritto del Terziario, 2

Cattaneo O., G. Gereffi, e C. Staritz (eds) (2010), Global value chains in a postcrisis world: A development perspective, Washington DC: The World Bank, 3-20, <http://issuu.com/world.bank.publications/docs/ 9780821384992>

Gallouj F. e F. Djellal (Eds.) (2010), The Handbook of Innovation and Services. A Multi-disciplinary Perspective, Edward Elgar, Cheltenham

Interbrand (2012), Best Global Brands 2012, Interbrand Report, $<$ http://www.interbrand.com/en/best-global-brands/2012/Best-GlobalBrands-2012.aspx>

McKinsey (2012), Manufacturing the Future: the next era of global growth and innovation, McKinsey Report, <http://www.mckinsey.com /insights/mgi/research/productivity_competitiveness_and_growth/the_f uture_of_manufacturing>

Miozzo M. e I. Miles (Eds.) (2002), Internationalization, Technology and Services, Edward Elgar, Cheltenham

Muller E. e A. Zenker (2001), "Business Services as Actors of Knowledge Transformation: The Role of Kibs in Regional and National Innovation Systems", Research Policy, 30 (9) 
Pine J.B. e J.H. Gilmore (2000), L'economia delle esperienze. Oltre il servizio, Etas, Milano

Ritzer G. (1997), Il mondo alla McDonald, il Mulino, Bologna

Rullani E. (2004), La fabbrica dell'immateriale. Produrre valore con la conoscenza, Carocci, Roma

Vandermerwe S. e J. Rada (1988), "Servitization of Business: Adding Value by Adding Services", European Management Journal, 6 